\title{
AJUSTE DE UN MODELO DE VOLUMEN DE ÁRBOL INDIVIDUAL DE Acacia mearnsii De Wild. CRECIENDO EN LA REGIÓN DEL BIO BIO
}

\author{
Pinilla. Juan Carlos ${ }^{1}$; Luengo, Karina; Navarrete, Mauricio y Navarrete, Felipe.
}

\section{RESUMEN}

Chile ha alcanzado un importante desarrollo forestal basado en la silvicultura y utilización de plantaciones de especies de rápido crecimiento, actualmente existen en el país 2,42 millones de hectáreas de plantaciones de Pinus radiata D. Don $(1,39)$, Eucalyptus globulus Labill. $(0,59)$, Eucalyptus nitens (Deane \& maiden) Maiden $(0,27)$ y otras especies $(0,17)$ (INFOR,2018), y una industria derivada de gran desarrollo en las áreas de aserrío, pulpa y papel, tableros y chapas, y diversos otros productos.

Entre las otras especies se encuentra diversas especies de los géneros Acacia, Atriplex, Eucalyptus, Pinus, Pseudotsuga, Populus y otros, introducidas al país en diferentes momentos. principalmente por el Instituto Forestal (INFOR).

Respecto de las plantaciones, sin embargo, existen en diferentes regiones del país condiciones climáticas y suelos forestales en los que el cultivo de las especies habitualmente empleadas no ha tenido los resultados esperados y se hacen necesarias otras especies.

Se suman los efectos del cambio climático que, por variaciones en los regímenes de precipitaciones y temperaturas, pueden tornar marginales algunas zonas del país para el crecimiento de estas especies tradicionalmente usadas en las plantaciones.

Se haría así prácticamente ineludible diversificar las especies forestales en las plantaciones, como medio para enfrentar las limitantes que impondrá el cambio climático y para cubrir nuevos sitios hoy no apropiados para las especies en uso.

Dentro de este marco INFOR ha desarrollado líneas de investigación sobre nuevas especies y su manejo, con el fin de ofrecer alternativas para los propietarios y comunidades rurales con nuevas opciones productivas. En estas líneas de trabajo se ha incluido numerosas especies de distintos géneros, entre estas especies australianas del género Acacia, como Acacia dealbata Link., Acacia melanoxylon R. Br. y Acacia mearnsii de Wild., de buena adaptación y crecimiento en diferentes sitios del país.

Comprobada la buena adaptación de nuevas especies se hace necesario el desarrollo de herramientas de apoyo para su manejo, como funciones de volumen y modelos predictivos de crecimiento y productividad.

En el presente trabajo de describe la elaboración de un primer modelo de volumen de árbol individual para la especie acacia negra (Acacia mearnsii) a partir de información obtenida de un rodal en crecimiento en la comuna de Florida, Región del Bio Bio, los principales aspectos de la metodología utilizada y los resultados obtenidos.

Palabras clave: Plantaciones forestales, Acacia mearnsii, función de volumen.

${ }^{1}$ Investigadores Instituto Forestal, Chile. jpínilla@infor.cl 


\section{SUMMARY}

Chile has an important forest development based on the silviculture and utilization of fast growing species planted forests, currently the country has a total planted area of 2.42 million hectares; Pinus radiata D. Don (1.39), Eucalyptus globulus Labill. (0.59), Eucalyptus nitens (Deane \& maiden) Maiden $(0.27)$ and other species $(0,17)$, and a well-developed forest industry on the sawnwood, cellulose and paper, boards and veneers, and other different production areas.

Among other species there are several species of the genera Acacia, Atriplex, Eucalyptus, Pinus Pseudotsuga, Populus and other, introduced to the country in the past, meanly by the Forestry Institute (INFOR).

Regarding to the planted forests however, there are zones in the country where climatic and soil conditions are not appropriated to establish and growth planted forests with the usual species and new species become necessary. Besides that, the climate change effects, including rainfall and temperature variation, can turn inappropriate other areas. Accordingly, seems to be inescapable a species diversification in planted forests as a mean to face the climate change effects and to cover new sites.

Under the mentioned framework, INFOR has carried out different research line on new species and its silviculture and management in order to offer some alternatives to the owners and rural communities with new productive options. The research lines have included a number of species and among them Australian species of the Acacia genus such as Acacia dealbata Link., Acacia melanoxylon $\mathrm{R}$. Br. And Acacia mearnsii De Wild., with a good adaptation and growth in different sites along the country.

Confirmed the adaptation of new species become necessary the development of management tools, such as volume functions and growth and productivity predictive models.

In this paper it is described the development of a first individual tree volume model for Black Wattle (Acacia mearnsii) based on information from a stand located at the Florida Commune, Bio Bio Region, as well as the methodology and the results.

Key words: Planted forests, Acacia mearnsii, Volume function. 


\section{INTRODUCCIÓN}

El sector forestal chileno se caracteriza por un importante desarrollo silvicultural e industrial basado en plantaciones de especies exóticas de rápido crecimiento, principalmente Pinus radiata y Eucalyptus spp. Sin embargo, existen condiciones climáticas y suelos forestales en los que el cultivo de estas especies no ha tenido los resultados esperados y se hacen necesarias otras especies. Se suman a esto los efectos del cambio climático que, por variaciones en los regímenes de precipitaciones y temperaturas, podrá tornar marginales algunas zonas del país para el crecimiento de estas especies tradicionalmente usadas en las plantaciones.

Se haría así ineludible diversificar las especies forestales en las plantaciones, como medio para enfrentar las limitantes que impondrá el cambio climático, para cubrir nuevos sitios hoy no apropiados para las especies en uso e incluso para repoblar sitios específicos y diferenciar productos. Se busca con esto una mejor adaptación a los sitios, apoyar procesos de forestación y reforestación, mantener o elevar la productividad de los predios, diversificar los productos y atenuar potenciales problemas fitosanitarios, entre otros aspectos. Dentro de este marco el Instituto Forestal de Chile (INFOR) ha desarrollado líneas de investigación sobre nuevas especies y su manejo, modelos operacionales y técnicas silvícolas cuyos resultados o avances pueden aplicar los propietarios y comunidades rurales obteniendo nuevas opciones productivas para incrementar su competitividad y la del sector.

En materia de nuevas especies, INFOR desde sus inicios, a principios de los años 60 del siglo pasado, ha desarrollado múltiples investigaciones que han incluido diversos géneros, orígenes y especies, entre ellas varias de origen australiano del género Acacia y una de ellas, con un interesante potencial para diferentes zonas del país, es acacia negra (Acacia mearnsii), especie que también ha sido introducida en América del Norte y del Sur, en Asia, en Europa, en África y en Nueva Zelanda, en distintas condiciones de sitio. Destacan sus usos a nivel internacional para la obtención de celulosa, de biomasa para energía y también de taninos a partir de su corteza, entre otros.

INFOR ha establecido diversos ensayos con esta especie en el país, abarcando diferentes situaciones de sitio, para generar información en cuanto a su adaptación y crecimiento en cada una de estas condiciones. Los resultados obtenidos, señalan que a los 8 años de edad es posible alcanzar valores promedio de $13 \mathrm{~cm}$ y $13 \mathrm{~m}$ en DAP y altura, respectivamente, con máximos de $18 \mathrm{~cm}$ y $20 \mathrm{~m}$, para DAP y altura, respectivamente, con un área basal de $15 \mathrm{~m}^{2} / \mathrm{ha}$ y una supervivencia de un $90 \%$. Estos resultados demuestran que Acacia mearnsii presenta un buen crecimiento en Chile, lo que la constituye en un recurso interesante, tanto para la industria forestal como para la pyme y los pequeños y medianos propietarios, siendo una alternativa posible de utilizar tanto por la pyme industrial como por pequeños y medianos propietarios forestales.

Se trabaja ahora en el análisis de la información reunida en los ensayos con el fin de obtener mayor conocimiento en lo relacionado con la estimación de rendimiento y crecimiento de la especie y desarrollar modelos matemáticos de manejo forestal que predigan producciones volumétricas y rendimientos de esta acacia en plantaciones forestales. En este trabajo se presentan los resultados del proceso de ajuste y validación de un modelo de volumen de árbol individual para la especie a partir de la información derivada de un ensayo establecido el año 2014 en la comuna de Florida, Región del Bio Bio, con el objetivo de generar modelos útiles para la gestión y manejo forestal de este tipo de plantaciones.

Para la adecuada utilización de nuevas especies en el sector forestal nacional se requiere desarrollar y promover la utilización de las especies y de las herramientas de apoyo para su cultivo y manejo. Al respecto, los modelos predictivos de crecimiento y productividad son básicos para una adecuada orientación en la toma de decisiones. Se trata así de generar y validar los modelos de crecimiento y rendimiento, en su adaptabilidad o funcionalidad para la especie; funciones de volumen de árbol individual y funciones predictivas de área basal, mortalidad y altura dominante.

Por esta razón, se realiza un estudio para generar un primer modelo de volumen de árbol individual para la especie Acacia mearnsii a partir de información obtenida de un rodal en 
crecimiento en la comuna de Florida, Región del Bio Bio.

El presente documento entrega los principales aspectos de la metodología utilizada en la toma de información desde el rodal, el uso de la información para evaluar la aplicabilidad de modelos de volumen anteriormente desarrollados por INFOR y posibles de utilizar para otras especies forestales (Bahamondez et al., 1995), y los resultados acerca de la funcionalidad del modelo obtenido.

\section{OBJETIVOS}

El objetivo de este estudio es generar, evaluar y difundir información sobre modelos de estimación de crecimiento, para orientar el desarrollo y manejo de plantaciones forestales de Acacia mearnssi, en aspectos relacionados con su productividad, potencial económico de cultivo e identificación de nuevas opciones productivas, consideradas estratégicas para incrementar la competitividad sectorial.

\section{LA ESPECIE}

Pinilla (2000) señala que Acacia mearnsii es originaria del sudeste de Australia, encontrándose principalmente en las tierras bajas y costeras y en las laderas bajas de las mesetas y cordilleras. Se distribuye desde el nivel del mar hasta aproximadamente los $900 \mathrm{msnm}$. Recibió su nombre botánico en honor al colector del espécimen tipo, el cirujano americano E. A. Mearns (1856-1916). El nombre común, Black Wattle, aromo negro o acacia negra, se refiere al follaje y a la corteza, ambos de color oscuro.

Acacia mearnsii es un arbusto grande o un árbol pequeño, comúnmente de 6 a $10 \mathrm{~m}$., pero puede alcanzar hasta $20 \mathrm{~m}$. de altura (Boland et al., 1984; Turnbull, 1986, Cit. por Kannegiesser, 1990).

Los ejemplares que crecen solitarios presentan copas amplias y extendidas, son ramificados desde la base y con el tronco principal torcido. Pero al crecer en plantaciones, el fuste es generalmente recto y delgado hasta tres cuartas partes de la altura total (Figura $N^{\circ} 1$ ).

La corteza de los árboles viejos es de color café negruzco, dura y fisurada, pero en individuos más jóvenes y en las partes altas de aquellos de más edad es de color café grisáceo, más delgada y suave.

El follaje adulto es de color verde oscuro con brotes nuevos suavemente amarillos. Su madera es finamente texturada y presenta anillos de crecimiento poco visibles. La albura es de color café muy pálido y el duramen, café claro con moteado rojizo. La durabilidad es considerada ligera o moderada. La densidad básica es de aproximadamente $630 \mathrm{~kg} / \mathrm{m}^{3}$ y la densidad de la madera secada al aire fluctúa entre 550 y $800 \mathrm{~kg} / \mathrm{m}^{3}$ (Bootle, 1984. Cit. por Kannegiesser, 1990).

En Australia crece en las zonas climáticas húmedas y subhúmedas, cálidas y frías, con precipitaciones medias anuales de 625 a $900 \mathrm{~mm}$, que caen entre 105 a 175 días al año. Raramente se presenta en zonas donde las temperaturas exceden los $38^{\circ} \mathrm{C}$, no crece con temperaturas sobre $40^{\circ} \mathrm{C}$, y se reportan áreas en donde ha soportado hasta 40 días con heladas al año (Figura $N^{\circ}$ 2).

En cuanto a los suelos, se señala que esta acacia se ha encontrado en basaltos, granitos y areniscas, siendo común en suelos derivados de esquistos y pizarras metamórficas, aluviales y podzoles forestales profundos de moderada a baja fertilidad. En su lugar de origen, los mejores crecimientos se han reportado en suelos húmedos, relativamente profundos, de texturas livianas, bien drenados y generalmente ácidos, con un $\mathrm{pH}$ entre 5 y 6,5. $A$. mearnsii constituye el sotobosque de bosques altos y abiertos, dominados principalmente por Eucalyptus ovata, E. saligna, E. globulus, E. viminalis y E. radiata. En zonas donde coloniza tierras explotadas, suele formar rodales densos. 

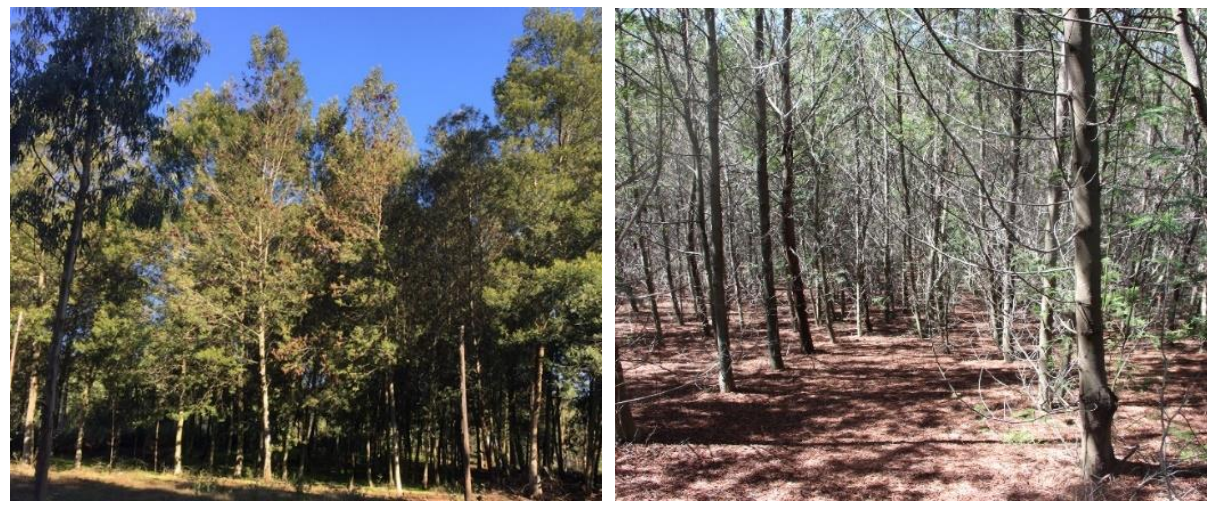

Figura $\mathrm{N}^{\circ} 1$

ÁRBOLES ADULTOS REGIÓN DEL BIO BIO

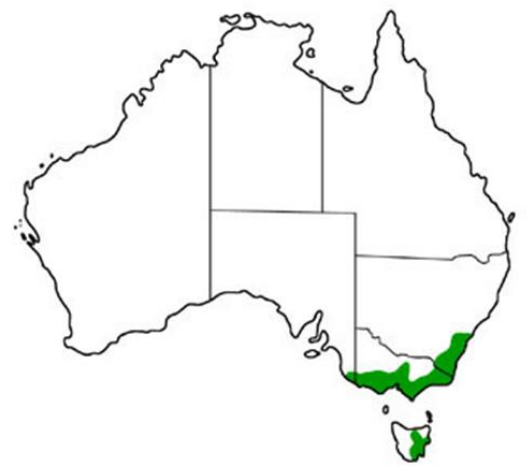

(Fuente: Australian Government, 2018)

Figura $\mathrm{N}^{\circ} 2$

DISTRIBUCIÓN NATURAL EN AUSTRALIA

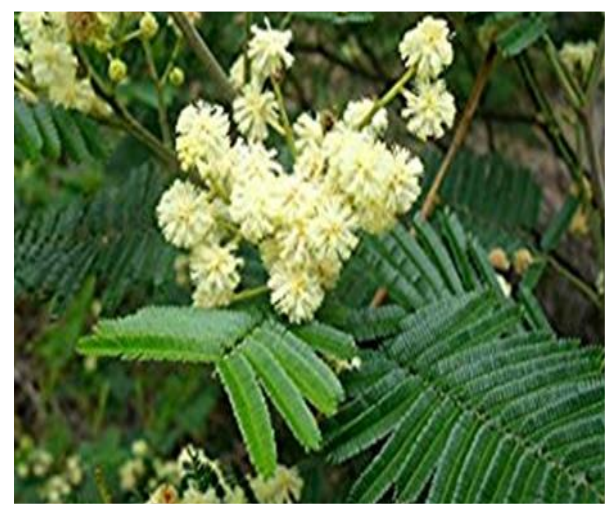

Figura $\mathrm{N}^{\circ} 3$

FOLLAJE Y FLORES 
La madera de esta especie se utiliza como material para construcciones livianas y biomasa para la generación de energía, pero posteriormente se comenzó a utilizar su corteza como materia prima para la curtiembre dado su alto contenido de taninos; 36 a $41 \%$ de taninos, excepcionalmente buenos, dependiendo los rendimientos de factores ambientales. Hoy es fuente principal de esta materia prima vegetal a nivel mundial para la manufactura de cuero duro para zapatos, monturas y otros productos. Las industrias de taninos basadas en esta especie se han desarrollado principalmente en Brasil, Kenya, India, Sudáfrica, Tanzania y Zimbabwe.

La literatura reporta otros usos para la corteza y el extracto obtenido, tales como preservantes, adhesivos, en molduras de plástico y como adhesivos en la industria de la madera prensada para exteriores, tableros de partículas y madera laminada (Coopens et al., 1980. Cit. por Kannegiesser, 1990). Se destaca que el adhesivo en base al extracto de corteza de esta especie presenta características de resistencia al agua similares a aquellos del tipo resorcinol, pero con un menor costo de producción.

La madera de $A$. mearnsii proporciona también leña de excelente calidad, con un peso específico de 0,70 a $0,85 \mathrm{~g} / \mathrm{cm}^{3}$ y un poder calorífico de 14.630 a $16.720 \mathrm{~kJ} / \mathrm{kg}$. Las dificultades en el secado y el agrietamiento de madera han disminuido su uso en la industria del mueble.

Por sus características de rápido crecimiento, adaptabilidad a diversas condiciones de sitio y capacidad colonizadora de áreas erosionadas, esta especie ha sido efectiva para el control de zonas degradadas y mejoramiento de la fertilidad de suelos, siendo una eficiente fijadora de nitrógeno. La literatura menciona que en Indonesia se han obtenido 21 a 28 t/año de hojas verdes, conteniendo 240 a $285 \mathrm{~kg}$ de nitrógeno y que cultivos agrícolas asociados a esta especie rinden el doble gracias a la acción fijadora del nitrógeno (NAS, 1980; Kannegiesser, 1990; Pinilla, 2000).

En estudios desarrollados por INFOR la especie se recomienda para las regiones de Valparaíso a Los Lagos y los productos a obtener son taninos extraíbles de su corteza y madera para uso en postes. Además, se indica que presenta una moderada resistencia a las heladas y que contribuye a la recuperación de sistemas degradados mediante el desarrollo de técnicas de control de erosión y restauración de la cubierta vegetal. En zonas de baja precipitación ha presentado problemas de crecimiento (INFOR, 1999; Pinilla, 2000).

Pinilla et al. (2018) informan sobre ensayos instalados por INFOR abarcando diferentes situaciones de sitio, con el propósito de determinar su respuesta a estas distintas condiciones, y agregan que estas investigaciones han demostrado que la especie presenta un adecuado crecimiento en el país.

Para determinar las condiciones en las cuales es posible el uso de las especies forestales, es necesario validar los diferentes aspectos técnicos y económicos en las distintas situaciones de crecimiento y mercado en el país.

\section{MATERIAL Y MÉTODO}

Para el estudio se seleccionó la unidad demostrativa y experimental de Acacia mearnsii establecida por INFOR en el sector de San Antonio de Cuda, Comuna de Florida, Región del Bio Bio, que tiene actualmente una edad de 14 años (Figura $N^{\circ} 4$ ).

La selección de los árboles para el estudio en este ensayo se realizó en base a la distribución diamétrica, realizándose un censo del $25 \%$ de la unidad, donde se encontraron 334 árboles y se seleccionaron 150 árboles, de los cuales 60 fueron utilizados en el estudio.

Luego de la selección de los árboles a utilizar, estos fueron medidos en pie y luego volteados registrándose diámetros con corteza a intervalos regulares a lo largo del fuste: 0,1 m, 1,3 $\mathrm{m}$ y cada $3,2 \mathrm{~m}$ hasta donde el diámetro del fuste mantenga un mínimo de $5 \mathrm{~cm}$ (Figura $\mathrm{N}^{\circ} 5$ ). 

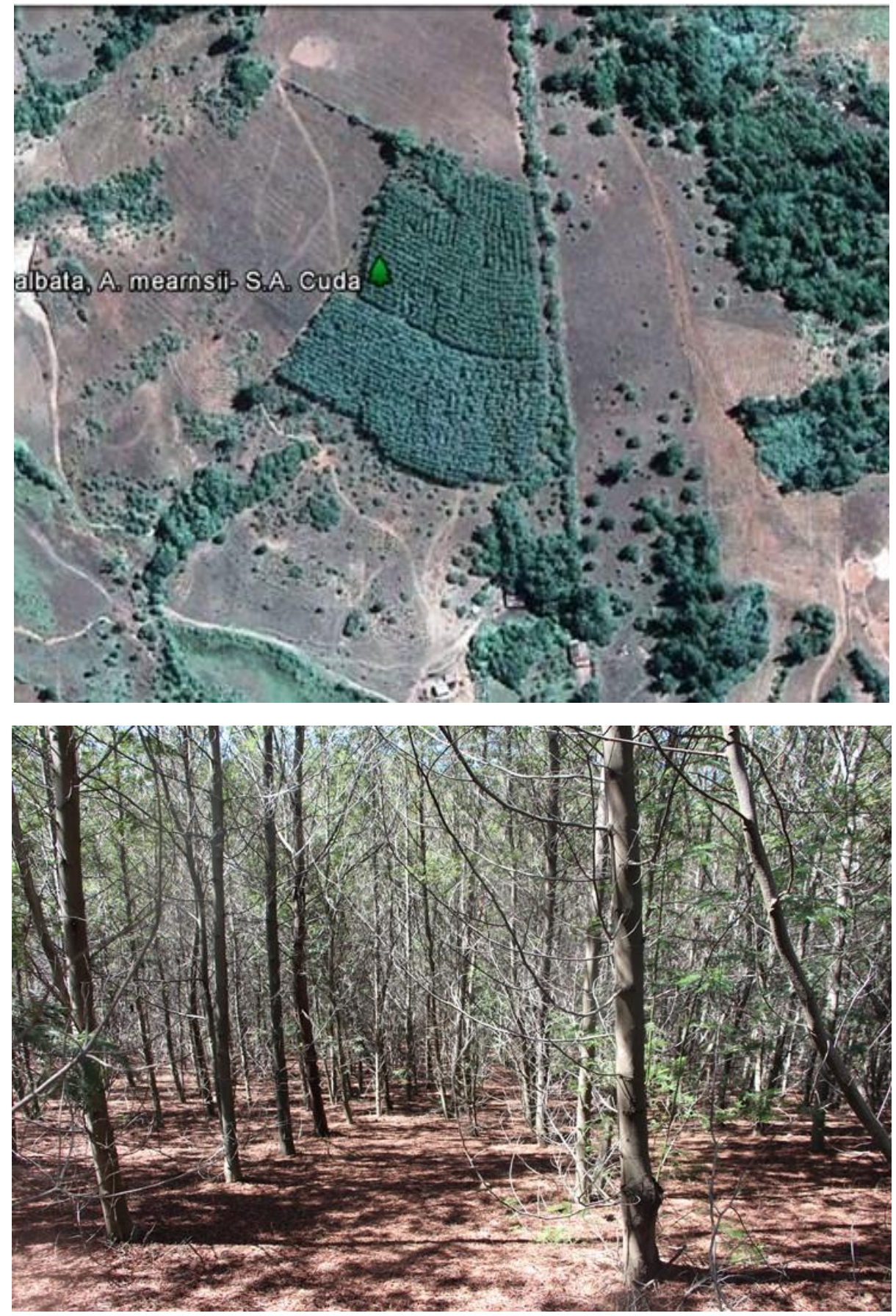

Figura $N^{\circ} 4$

UNIDAD EXPERIMENTAL SECTOR SAN ANTONIO DE CUDA, FLORIDA, REGIÓN DEL BIO BIO 

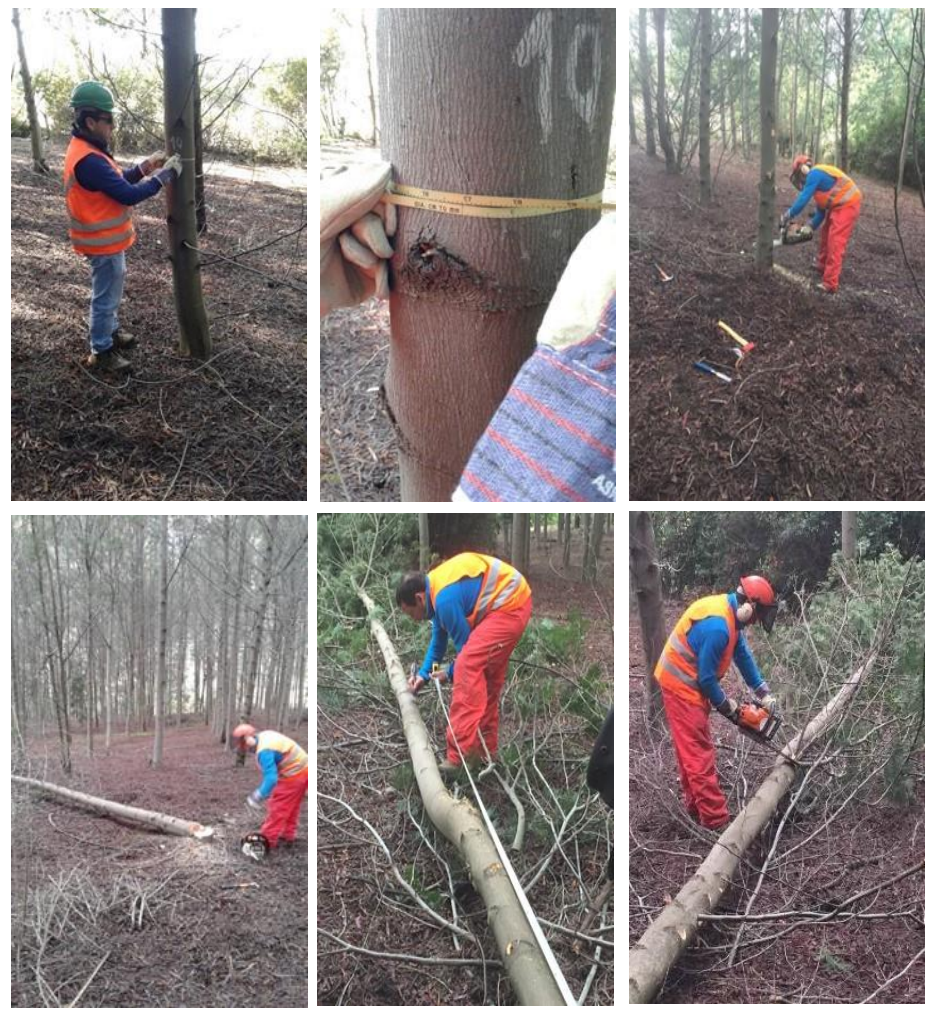

Figura $\mathrm{N}^{\circ} 5$

SELECCIÓN, VOLTEO Y MEDICIÓN DE ÁRBOLES

\section{Función de Volumen}

La determinación de los volúmenes de cada árbol se puede obtener a través de diferentes procedimientos prácticos y teóricos. Entre estos, se incluyen diámetros medidos a distintas alturas del fuste, ocupando factores de forma que modifiquen el volumen del cilindro teórico al que se asemeja, o a través de funciones de ahusamiento o por rotación de cuerpos simples.

Uno de los sistemas más usados es la cubicación por secciones obtenidas a distintas alturas del fuste. Los árboles muestras se cortan en largos relativos o absolutos y se cubican por trozas, donde la exactitud de las estimaciones por estas fórmulas depende del número de ellas. Las fórmulas de cubicación más conocidas son la de Smalian (igual a un paraboloide truncado) y la de Huber y Newton, entre otras (Prodan et al.., 1997). La fórmula de Smalian se define como:

$$
\text { Smalian : } v=\left(g_{b}+g_{s}\right) * \frac{l}{2}
$$

Donde: $\quad g_{b}, g_{s}$ : Áreas de la sección basal y superior de la troza.

$L \quad$ : Largo de la sección utilizada. 
Los volúmenes individuales por árbol fueron determinados por la acumulación de volúmenes de pequeñas secciones observadas e interpoladas calculados con Smalian. Los árboles que tenían más secciones observadas acumulaban un total de 27, estando la media cercana a las 12 secciones por árbol.

Luego el volumen de cada árbol se obtuvo mediante la acumulación de dichos volúmenes para el cálculo del volumen total comercial (hasta diámetros sin corteza de $5 \mathrm{~cm}$ ). Los volúmenes finalmente obtenidos se graficaron para observar su distribución según el DAP.

Dado el objetivo de este estudio, se analizó si la función de volumen de árbol individual tradicionalmente empleada, el modelo de variables combinadas (Spurr) (Bahamondez et al, 1995; Martin y Bahamóndez, 2000.), es compatible con Acacia mearnsii. La estructura de este modelo es la siguiente:

$$
\mathrm{Vol}=\mathrm{a}+\mathrm{b}^{\star} \mathrm{DAP}{ }^{2 *} \mathrm{H}
$$

Donde: Vol : Volumen individual en $\mathrm{m}^{3} \mathrm{ssc}$ para un $\mathrm{IU}=5 \mathrm{~cm}$

$\mathrm{a}, \mathrm{b} \quad$ : Coeficientes del modelo

DAP : Diámetro a la altura del pecho $(\mathrm{cm})$

$\mathrm{H} \quad$ : Altura total $(\mathrm{m})$

El modelo se ajusta por métodos de mínimos cuadrados a través de regresión ponderada por $\left(1 / \mathrm{DAP}^{2} \mathrm{H}\right)$, para evitar problemas de heterocedasticidad.

Al respecto, Friedl et al. (2010) mencionan que la ecuación logarítmica de variables combinadas de Spurr es el modelo más conveniente para la predicción de los volúmenes, con y sin corteza, de árboles individuales de Acacia mearnsii, utilizado en el sur de Brasil, lo cual ya había sido señalado por Schneider et al. (2000). Los modelos más utilizados corresponden a:

$$
\begin{aligned}
& \log v c c=b o+b 1{ }^{*} \log \left(d^{2} h / 10.000\right) \\
& \log v s c=b o+b 1{ }^{*} \log \left(d^{2} h / 10.000\right)
\end{aligned}
$$

Donde: $\quad v c c=$ Volumen total con corteza $\left(\mathrm{m}^{3}\right)$

$$
\begin{aligned}
& \mathrm{vsc}=\text { Volumen comercial sin corteza }\left(\mathrm{m}^{3}\right) \\
& \mathrm{d}=\text { Diámetro a la altura de pecho }(1,3 \mathrm{~m})(\mathrm{cm}) \\
& \mathrm{h}=\text { Altura total }(\mathrm{m})
\end{aligned}
$$

Otro modelo utilizado en Brasil para el volumen de árbol individual fue estimado por Schneider y Hosokawa (1978) y Schneider (1978), citados por Schneider et al. (2000), bajo la ecuación:

$$
\log v=-4,2007+0,9495 \times \log \left(d^{2} h\right)
$$

$$
\text { Donde: } \begin{aligned}
\quad & v=\text { Volumen con corteza }\left(\mathrm{m}^{3}\right) \\
\mathrm{d} & =\text { Diámetro a la altura del pecho }(\mathrm{cm}) \\
\mathrm{h} & =\text { Altura total }(\mathrm{m})
\end{aligned}
$$

\section{Evaluación de la Función de Volumen}

Como indicadores de la bondad de ajuste de la función de volumen se utilizaron: el RECM (\%) como estadístico de precisión y la DIFA (\%) de sesgo, ambos expresados como una proporción de la media del grupo, el coeficiente de correlación y el error estándar.

El Error Cuadrático Medio Porcentual (ECM \%) y la Diferencia Agregada Porcentual (DIFA \%) se definen matemáticamente por las siguientes expresiones: 


$$
\begin{aligned}
& \text { RECM } \%=\frac{100}{\bar{y}}\left[\frac{1}{n} \sum_{i=1}^{n}\left(y_{i}-\hat{y}_{i}\right)^{2}\right]^{1 / 2} \\
& D I F A \%=\frac{100}{\bar{y}}\left[\frac{1}{n} \sum_{i=1}^{n}\left(y_{i}-\hat{y}_{i}\right)\right]
\end{aligned}
$$

Donde: $\quad y_{i} \hat{y}_{i}$ : Corresponden al valor observado y estimado de la medición i-ésima;

$\bar{y} \quad$ : Es el promedio

$n \quad$ : Total de observaciones.

Para estos dos estadísticos, el mejor modelo corresponderá a aquel que presente los valores más cercanos a cero.

\section{RESULTADOS}

\section{Parámetros Generales de Rodal}

Como se mencionó previamente, se realizó una evaluación de los árboles de la especie para caracterizar su crecimiento, lo que permite a futuro relacionar parámetros descriptivos del crecimiento (DAP, altura) con variables de interés, como es el volumen de árbol individual y también la cantidad de materia seca. Los antecedentes recopilados permitieron la generación de herramientas de estimación del crecimiento y del volumen de árbol individual, para su uso posterior en la generación de antecedentes de productividad.

Es así como se obtuvo un diámetro promedio de los árboles de $14,9 \mathrm{~cm}$, mientras que la altura registró un valor medio de 17,1 m. La relación Diámetro-Altura observada en los árboles de Acacia mearnsii se presenta en el gráfico de la Figura $\mathrm{N}^{\circ} 6$, mientras que la frecuencia relativa de los diámetros se presenta el gráfico de la Figura $\mathrm{N}^{\circ} 7$. La descripción de los árboles utilizados en la muestra se presenta en los Cuadros $\mathrm{N}^{\circ} 1, \mathrm{~N}^{\circ} 2$ y $\mathrm{N}^{\circ} 3$.

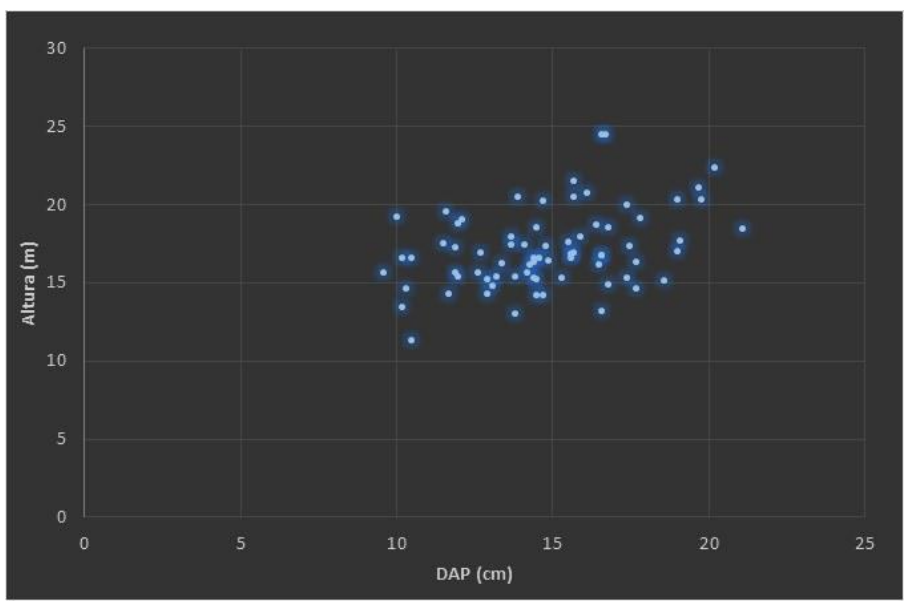

Figura $N^{\circ} 6$

RELACIÓN DIÁMETRO-ALTURA 

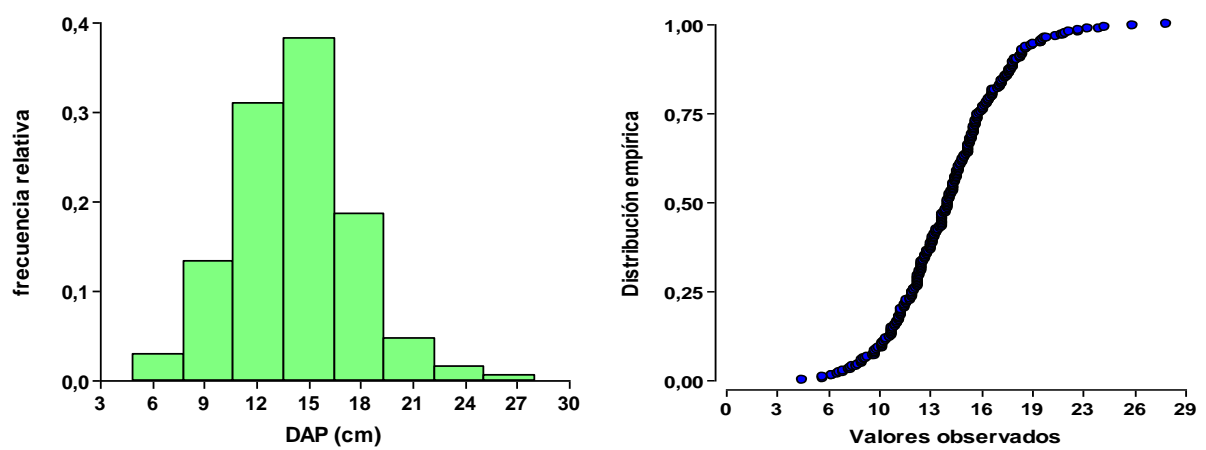

Figura $\mathrm{N}^{\circ} 7$

FRECUENCIA RELATIVA DE LOS DIÁMETROS OBSERVADOS EN TERRENO

Una vez obtenida la frecuencia de los árboles, se seleccionó una muestra de ellos para ser utilizada en el estudio para generar el modelo de volumen de árbol individual.

La selección de las muestras se realizó considerando la distribución diamétrica existente en el rodal, utilizando los rangos correspondientes al 25,50 y $75 \%$ de los valores obtenidos del diámetro. Al obtener estos valores se ubicaban en el rodal los árboles con estos diámetros para posteriormente ser volteados. De esta forma se seleccionaron finalmente 58 árboles para la realización del estudio.

Cuadro $\mathrm{N}^{\circ} 1$

DESCRIPCIÓN MUESTRA UTILIZADA

\begin{tabular}{|l|r|r|}
\hline Resumen & \multicolumn{1}{|c|}{$\begin{array}{c}\text { DAP } \\
\text { (cm) }\end{array}$} & $\begin{array}{c}\text { Altura } \\
(\mathbf{m})\end{array}$ \\
\hline$N^{\circ}$ casos & 58 & 58 \\
\hline$N^{\circ}$ de observaciones & 6 & 326 \\
\hline$N^{\circ}$ de observaciones/árbol & 14,21 & 6 \\
\hline Valor Medio & 1,68 & 15,18 \\
\hline Desviación estándar & 11,70 & 1,64 \\
\hline Valor mínimo & 16,90 & 12,25 \\
\hline Valor máximo & 14,20 & 18,90 \\
\hline Mediana & & 15,26 \\
\hline
\end{tabular}

Cuadro $\mathrm{N}^{\circ} 2$

DESCRIPCIÓN MUESTRA UTILIZADA

\begin{tabular}{|c|c|c|c|c|c|c|c|c|}
\hline Variable & Casos & Observaciones & $\begin{array}{c}\text { Observaciones } \\
\text { por árbol }\end{array}$ & $\begin{array}{c}\text { Valor } \\
\text { Medio }\end{array}$ & $\begin{array}{c}\text { Desviación } \\
\text { Estándar }\end{array}$ & $\begin{array}{c}\text { Valor } \\
\text { Mínimo }\end{array}$ & $\begin{array}{c}\text { Valor } \\
\text { Máximo }\end{array}$ & Mediana \\
\hline DAP (cm) & 58 & 326 & 6 & 14,21 & 1,68 & 11,7 & 16,9 & 14,2 \\
\hline Altura (m) & 58 & 326 & 6 & 15,18 & 1,64 & 12,25 & 18,9 & 15,26 \\
\hline
\end{tabular}


Cuadro $\mathrm{N}^{\circ} 3$

DESCRIPCIÓN MUESTRA SEGÚN CLASE DIAMÉTRICA Y RANGOS DE ALTURA

\begin{tabular}{|c|c|c|c|c|c|c|}
\hline $\begin{array}{l}\text { Clase } \\
\text { DAP } \\
(\mathrm{cm}) \\
\end{array}$ & $\begin{array}{c}\text { Árboles } \\
\left(\mathrm{N}^{\circ}\right)\end{array}$ & $\begin{array}{c}\text { DAP } \\
\text { Mínimo } \\
(\mathrm{cm})\end{array}$ & $\begin{array}{l}\text { DAP } \\
\text { Máximo } \\
\text { (cm) }\end{array}$ & $\begin{array}{c}\text { Altura } \\
\text { Mínima } \\
(\mathrm{m})\end{array}$ & $\begin{array}{c}\text { Altura } \\
\text { Máxima } \\
\text { (m) }\end{array}$ & $\begin{array}{l}\text { Mediana } \\
\quad(\mathrm{m})\end{array}$ \\
\hline 11 & 15 & 11,7 & 11,9 & 12,40 & 16,69 & 14,39 \\
\hline 12 & 5 & 12,1 & 12,8 & 12,40 & 15,51 & 15,22 \\
\hline 13 & 14 & 13,8 & 13,9 & 12,25 & 17,33 & 16,02 \\
\hline 14 & 5 & 14,0 & 14,7 & 12,96 & 18,21 & 14,56 \\
\hline 15 & 12 & 15,5 & 15,9 & 13,07 & 18,90 & 15,64 \\
\hline 16 & 7 & 16,0 & 16,9 & 14,90 & 17,41 & 15,90 \\
\hline
\end{tabular}

En el gráfico de la Figura $N^{\circ} 8$ se presenta la distribución de frecuencias según clases de diámetro, en la Figura $N^{\circ} 9$ los valores medios en diámetro y altura de los árboles, y en la Figura $\mathrm{N}^{\circ}$ 10 la distribución de altura según clase de DAP para la muestra volumétrica.

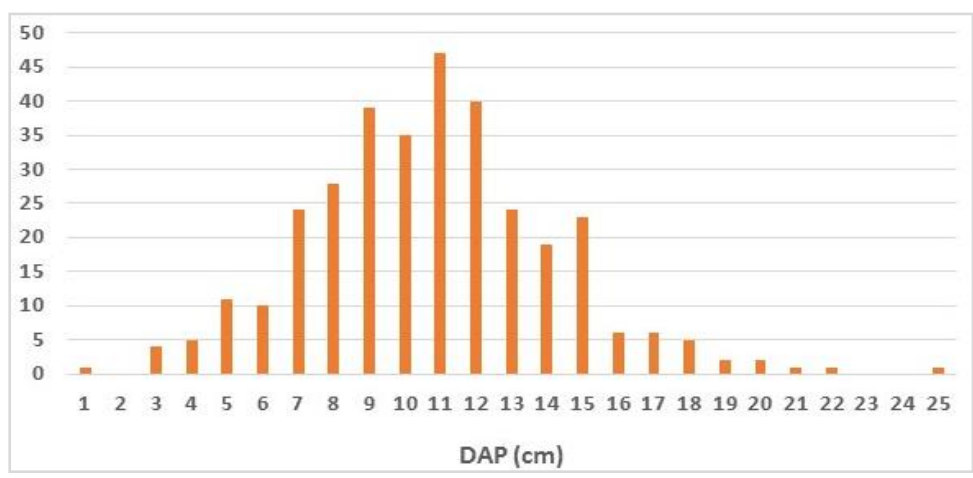

Figura $\mathrm{N}^{\circ} 8$

DISTRIBUCIÓN DE FRECUENCIAS SEGÚN CLASE DE DAP

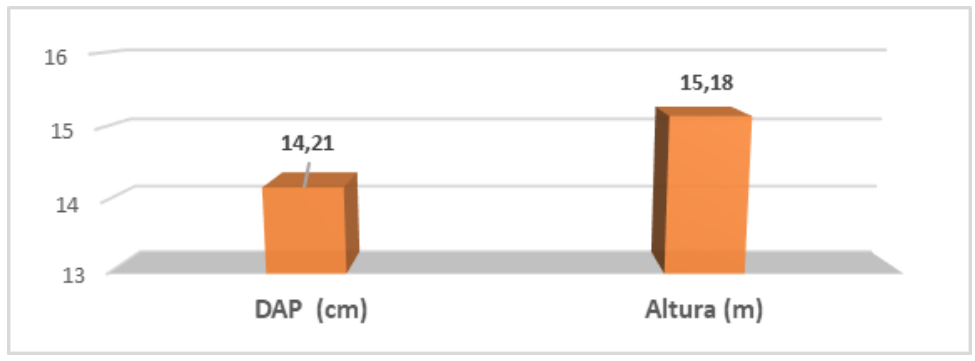

Figura $\mathrm{N}^{\circ} 9$

VALORES MEDIOS EN DIÁMETRO Y ALTURA DE LOS ÁRBOLES 


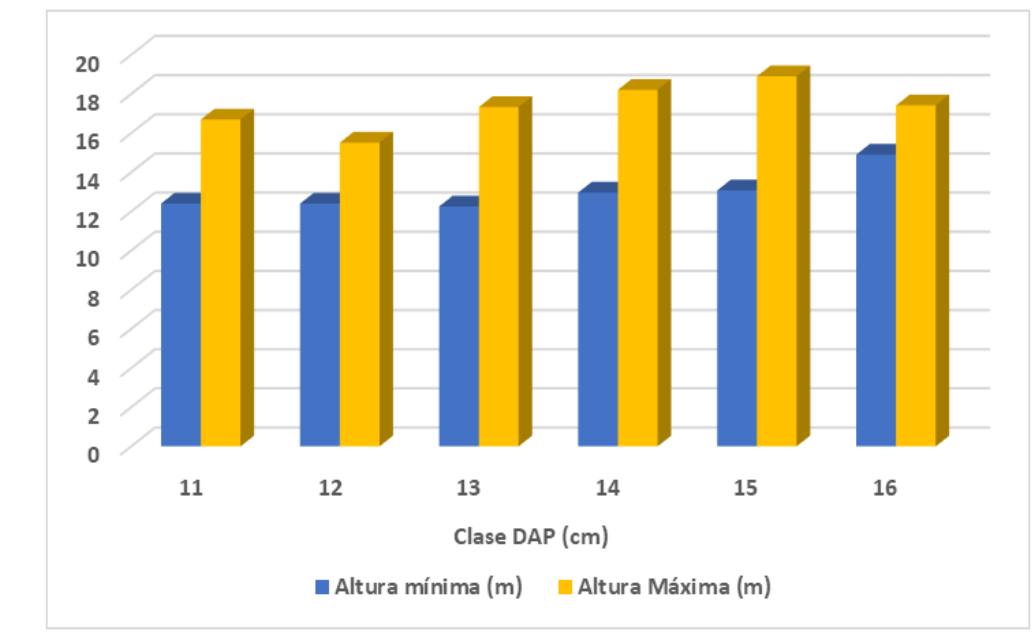

Figura $N^{\circ} 10$

DISTRIBUCIÓN DE ALTURAS SEGÚN CLASE DE DAP PARA LA MUESTRA VOLUMÉTRICA

\section{Aplicación y Selección del Modelo de Volumen de Árbol Individual}

A cada árbol se le calculó su volumen total, a través de la sumatoria del volumen de las trozas o secciones individuales, mediante el método de Smalian (V= [(Área sección inicial + Área sección final) / 2]* Largo troza).

DAP.

En la Figura $N^{\circ} 11$ se presentan los volúmenes de los árboles individuales en relación al

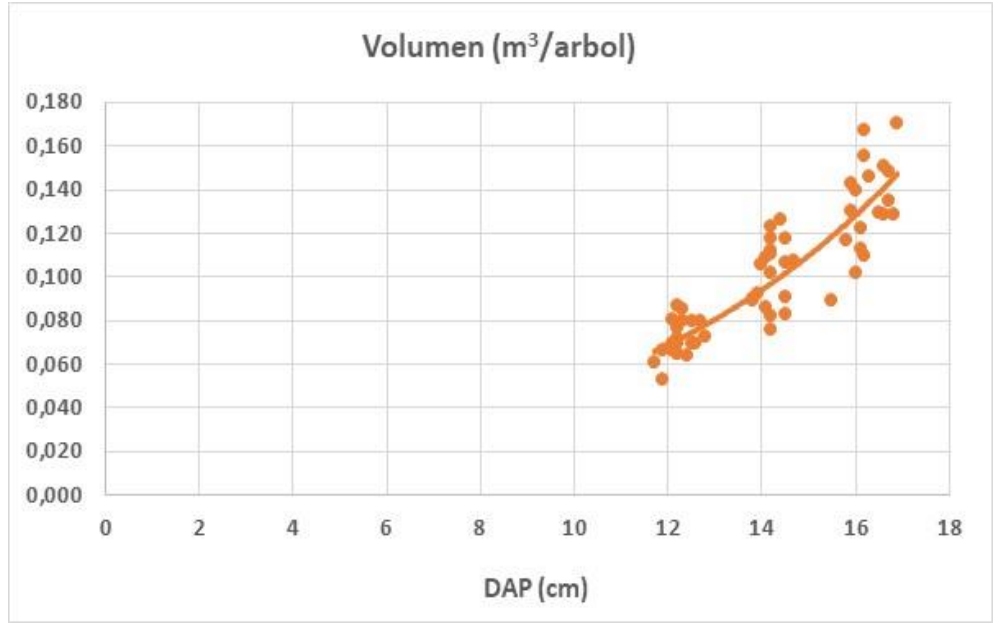

Figura $N^{\circ} 11$

VOLUMEN DE ÁRBOL INDIVIDUAL 
Se generó una base de datos que contenía los parámetros y volumen calculado de cada árbol, a la cual se agregaron las transformaciones de las variables principales (DAP, altura) de modo de obtener todas las combinaciones que correspondieran a las ya descritas como componente de los modelos de volumen indicados. La base de datos resultado de este proceso fue utilizada para realizar los procesos de análisis de regresión.

Una vez realizados los ajustes correspondientes y analizado los resultados, fue posible a través del método de los mínimos cuadrados y proceso de selección Stepwise, generar un set de modelos de volumen de árbol individual para Acacia mearnsii creciendo en la Región del Bio Bio.

Como primer resultado se puede señalar que el proceso no registró un buen ajuste de la función de volumen de árbol individual correspondiente al modelo de variables combinadas de Spurr.

Los modelos que generaron los mejores resultados y sus coeficientes de correlación se presentan en el Cuadro $\mathrm{N}^{\circ} 4$.

Cuadro $\mathrm{N}^{\circ} 4$

MODELOS OBTENIDOS Y SUS COEFICIENTES DE CORRELACIÓN

\begin{tabular}{|c|lc|c|}
\hline Modelo & \multicolumn{1}{|c|}{ Expresión } & $\mathbf{R}^{2}$ \\
\hline 1 & $\mathrm{VOL}=$ & $0,01+0,000039^{*} \mathrm{DAP}{ }^{2} \mathrm{H}-0,00015^{*} \mathrm{DAP}{ }^{2}$ & 0,9577 \\
\hline 2 & $\mathrm{VOL}=\quad 0,22984+0,00003^{*} \mathrm{DAP}{ }^{2} \mathrm{H}+0,00027^{*} \mathrm{DAPH}-0,08205^{*} \mathrm{LOGDAP}^{2} \mathrm{H}$ & 0,9581 \\
\hline 3 & $\mathrm{LOGVOL}=-3,429977+0,627478^{*} \mathrm{LOGDAP}{ }^{2} \mathrm{H}+1,09 \mathrm{E}-03^{*} \mathrm{DAPH}$ & 0,9594 \\
\hline
\end{tabular}

$\mathrm{VOL}=$ Volumen de árbol individual $\left(\mathrm{m}^{3} \mathrm{ssc}\right)$ hasta diámetro límite $5 \mathrm{~cm}$

DAP = Diámetro a la altura del pecho $(\mathrm{cm})$

$\mathrm{H} \quad=$ Altura total del árbol $(\mathrm{m})$

Al aplicar los indicadores de la bondad de ajuste de la función de volumen resultante, se obtuvieron los resultados que se presentan en el Cuadro $\mathrm{N}^{\circ} 5$.

\section{Cuadro $\mathrm{N}^{\circ} 5$}

INDICADORES DE LA BONDAD DE AJUSTE

PARA EL MODELO DE VOLUMEN DE ÁRBOL INDIVIDUAL

\begin{tabular}{|c|c|c|c|}
\hline Modelo & $\begin{array}{c}\text { RECM } \\
\text { (\%) }\end{array}$ & $\begin{array}{c}\text { DIFA } \\
(\%)\end{array}$ & $\mathbf{R}^{\mathbf{2}}$ \\
\hline 1 & 5,9804 & $-0,2761$ & 0,9577 \\
\hline 2 & 7,3619 & 4,1921 & 0,9581 \\
\hline 3 & 49,3591 & 35,7476 & 0,9594 \\
\hline
\end{tabular}

Luego de analizar los resultados obtenidos, es posible concluir que el modelo 1 presentó las mejores capacidades estimadoras del volumen de árbol individual. El modelo resultante es:

$$
\text { VOL }=0,01+0,000039^{*} D A P^{2} H-0,00015^{\star} D A P^{2}
$$

Donde: VOL = Volumen árbol individual $\left(\mathrm{m}^{3} \mathrm{ssc}\right)$ hasta un diámetro límite de $5 \mathrm{~cm}$

DAP = Diámetro a la altura del pecho $(\mathrm{cm})$

$\mathrm{H}=$ Altura total del árbol $(\mathrm{m})$ 


ANÁLISIS DE VARIANZA
\begin{tabular}{|l|c|c|c|c|c|}
\hline \multicolumn{1}{|c|}{ Fuente } & $\begin{array}{c}\text { Suma de } \\
\text { Cuadrados }\end{array}$ & g.I. & $\begin{array}{c}\text { Cuadrados } \\
\text { Medios }\end{array}$ & F & P \\
\hline Regresión & 0,851313791 & 1 & 0,851313791 & $1.06527 \mathrm{E}+04$ & 0.00 \\
\hline Residual & 0,005594066 & 70 & 0,000079915 & & \\
\hline Total & 1 & 71 &
\end{tabular}

Al aplicar el modelo obtenido en relación con los datos reales del volumen del árbol individual se genera el gráfico de la Figura $\mathrm{N}^{\circ} 12$.

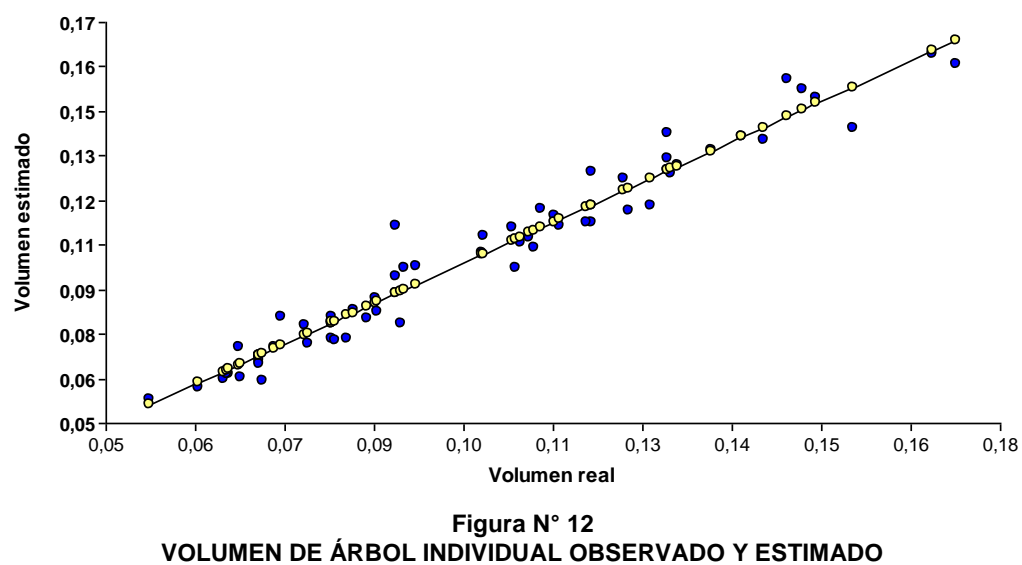

La bondad del ajuste del modelo puede ser también apreciada a través de la regresión hecha entre los valores reales del volumen y los valores estimados con el modelo utilizado. Los coeficientes de regresión obtenidos deben cumplir con ciertas hipótesis para asegurar que el ajuste es exacto y preciso, sin presentar sesgos.

La regresión se realiza ajustando un modelo lineal del tipo:

$$
\mathrm{Ve}=\mathrm{a}+\mathrm{b}^{*} \mathrm{Vo}_{0}
$$

Donde: $\begin{array}{ll}\mathrm{Ve} & =\text { Volumen estimado. } \\ \mathrm{Vo} & =\text { Volumen observado. } \\ \mathrm{a}, \mathrm{b} & =\text { Coeficientes de la regresión. }\end{array}$

Si en las regresiones obtenidas el valor de la constante (a) es igual a cero y el valor del término que acompaña a la variable independiente (b) es igual a 1 , se puede determinar que los ajustes son exactos y precisos. Esto significaría que existe una perfecta correspondencia entre el volumen estimado y el volumen real.

Para obtener la significancia estadística de los coeficientes de estas regresiones, los valores resultantes de las dócimas de hipótesis $\left(t_{a}=0 ; t_{b}=1\right)$ se deben comparar con los tabulares de la distribución $t$ de Student, fijando una región critica para una probabilidad $a=0,05$, con 
contraste bilateral. Si el valor calculado es menor que el valor critico de t para los correspondientes grados de libertad, (n-2), se infiere que la estimación proporciona la exactitud requerida.

Los coeficientes del modelo así resultante y su significación estadística para este análisis corresponden a:

$$
\mathrm{Ve}=0,000065+0,996585^{\star} \mathrm{Vo}_{0}
$$

Con: $\quad R^{2}: 0,97$

Error estándar de estimación: 0,00894

El análisis de varianza obtenido se indica en el Cuadro $N^{\circ} 7$.

Cuadro $\mathrm{N}^{\circ} 7$

ANÁLISIS DE VARIANZA

\begin{tabular}{|c|c|c|c|c|c|}
\hline $\begin{array}{c}\text { Fuente } \\
\text { de } \\
\text { Variación }\end{array}$ & $\begin{array}{c}\text { Suma de } \\
\text { Cuadrados }\end{array}$ & g.l. & $\begin{array}{l}\text { Cuadrados } \\
\text { Medios }\end{array}$ & $\mathbf{F}$ & $P$ \\
\hline Modelo & 0,048220 & 1 & 0,048220 & $1.267,319963$ & $<0,0001$ \\
\hline Vol Estimado & 0,048220 & 1 & 0,048220 & $1.267,319963$ & $<0,0001$ \\
\hline Error & 0,002131 & 56 & 0,000038 & & \\
\hline Total & 0,050350 & 57 & & & \\
\hline
\end{tabular}

El análisis estadístico permite asegurar que el valor de "b" es similar a 1, y el valor de "a" similar a 0 . La ausencia de sesgos importantes permite apreciar las buenas propiedades estimadoras de los modelos ajustados.

El análisis de varianza señala, además, que la función de volumen obtenida explica muy bien los datos, ya que el valor del $F_{\text {cal }}$ es mayor que el $F_{\text {tabla }}$ por lo que las variables independientes tienen influencia en la variable dependiente, haciendo estadísticamente significativo el modelo obtenido.

El modelo presenta un ajuste apropiado para los datos y, dada su simplicidad, se selecciona este modelo como el más aconsejable.

\section{ANÁLISIS DE RESULTADOS}

La cuantificación del volumen de los árboles individuales y las plantaciones forestales es una de las principales variables a considerar para la caracterización y descripción de los bosques, en donde este valor representa el resultado de una serie de factores asociados principalmente al sitio forestal, la carga genética y el manejo realizado, que se expresa a través del desarrollo de las variables del diámetro y la altura.

Se ha facilitado la estimación de este parámetro a través del desarrollo y utilización de modelos estadístico-matemáticos que utilizan como variables predictoras del volumen parámetros del árbol de rápida medición, como son el DAP y la altura total y/o alguna otra variable.

Este tipo de modelos predictivos ha sido muy utilizado en el país para su aplicación en las especies forestales de mayor uso en el sector forestal chileno, como lo es para el caso de pino radiata y algunas especies de eucalipto.

Sin embargo, importantes esfuerzos de diversificación se están desarrollando en el país, para enfrentar de los efectos del cambio climático, la aparición de nuevas plagas o enfermedades 
forestales o para acceder a nuevos nichos de mercado. Para avanzar en la diversificación forestal se requiere, entre otros avances, del desarrollo de herramientas de estimación del crecimiento y productividad en diferentes situaciones de crecimiento y edades. Es necesario así generar nuevas herramientas de predicción del volumen del árbol individual, en este caso de la especie Acacia mearnsii, como una forma de proveer de antecedentes para el fomento a su utilización, siendo el volumen a generar el parámetro de mayor interés.

En este estudio, los valores registrados son similares a los indicados por la bibliografía, la que indica que Acacia mearnsii es un arbusto grande o un árbol pequeño, comúnmente de 6 a $10 \mathrm{~m}$ de altura, pero puede alcanzar hasta $20 \mathrm{~m}$ (Kannegiesser, 1990), antecedentes también corroborados por otros estudios de INFOR con la especie (Pinilla et al., 2015).

Pinilla et al. (2018) señalan que a los 8 años de edad se registran valores promedios de $13 \mathrm{~cm}$ y $13 \mathrm{~m}$ en DAP y altura, respectivamente, con máximos promedio de $18 \mathrm{~cm}$ y $20 \mathrm{~m}$, para el DAP y altura, respectivamente, con un área basal de $15 \mathrm{~m}^{2} /$ ha y supervivencia de un $90 \%$.

La investigación de INFOR con Acacia mearnsii señala que la especie presenta un adecuado crecimiento en Chile, similar a otros países donde se la cultiva, lo que la transforma en un recurso interesante para la industria forestal local, en especial para pequeños y medianos propietarios forestales.

El trabajo desarrollado por INFOR es un esfuerzo por lograr información confiable y certera sobre el crecimiento de la especie, el que se ha complementado con estudios de las propiedades de su madera y con algunos primeros avances en un programa de mejoramiento genético.

En este estudio, los valores obtenidos con los ajustes al modelo utilizado, al aplicar la función obtenida para árboles de Acacia mearnsii, comparando los volúmenes reales medidos con los correspondientes estimados, permiten concluir que el modelo desarrollado, que utiliza como variables predictoras el DAP y la altura de los árboles, parece representar razonablemente bien el comportamiento volumétrico de los árboles de esta especie.

La bondad del ajuste del modelo y la ausencia de sesgos importantes entre los valores reales y los estimados, confirma las buenas propiedades estimadoras del modelo ajustado.

El análisis realizado se enmarca en el estudio de la aplicabilidad de los modelos sobre situaciones de crecimiento de la especie en la Región del Bio Bio, requiriéndose ahora validar este modelo en otras situaciones de crecimiento, o bien desarrollar nuevos ajustes y modelos de estimación, indispensables para su uso en la estimación del rendimiento de la especie.

\section{CONCLUSIONES}

Luego del análisis de los resultados obtenidos, se concluye que el modelo generado de volumen de árbol individual puede ser aplicado para la especie y que este tipo de información y herramientas de estimación debe apoyar el fomento a nuevas opciones silvícolas, permitiendo la estimación de su productividad y crecimiento en el país.

Los valores de crecimiento de los árboles de Acacia mearnsii en Florida, Región del Bio Bio, presentaron a los 14 años un diámetro promedio de $14,2 \mathrm{~cm}$, mientras que la altura registro un valor medio de $15,2 \mathrm{~m}$.

Los valores son similares a los indicados por la bibliografía y a otros estudios desarrollados por INFOR.

El proceso no registró un buen ajuste de la función de volumen de árbol individual correspondiente al modelo de variables combinadas de Spurr.

El volumen promedio de los árboles de Acacia mearnsii correspondió a 0,1014 m³ssc. 
Luego del análisis de la información el modelo de árbol individual que presentó las mejores capacidades estimadoras es:

$$
\text { VOL }=0,01+0,000039^{\star} D^{2} P^{2} H-0,00015^{\star} D^{2} P^{2}
$$

Donde: $\quad \mathrm{VOL}=$ Volumen árbol individual $\left(\mathrm{m}^{3} \mathrm{ssc}\right)$ hasta diámetro límite $5 \mathrm{~cm}$

DAP = Diámetro a la altura del pecho $(\mathrm{cm})$

$\mathrm{H} \quad=$ Altura total del árbol $(\mathrm{m})$

La bondad del ajuste del modelo y la ausencia de sesgos importantes entre los valores reales y los estimados, confirma las buenas propiedades estimadoras del modelo ajustado.

Acacia mearnsii se presenta como una especie promisoria por su adaptabilidad, características de crecimiento y posibles productos a obtener desde ella (madera, biomasa, taninos a partir de su corteza).

Los antecedentes de crecimiento indican que Acacia mearnsii es una alternativa atractiva para los forestadores e industrias del país.

Es necesario seguir obteniendo información del crecimiento de la especie en el país, así como continuar con el desarrollo de modelos locales de volumen abarcando otras áreas geográficas, considerando la posibilidad de ajustar funciones de biomasa.

Se hacen necesarias más investigaciones y por más tiempo, en relación a la especie, analizando especialmente los procesos de competencia por agua, luz y nutrientes.

\section{REFERENCIAS}

Australian Government, 2018. Acacia mearnsii. Department of Environment and Energy. In: http://www.environment.gov.au/cgi-bin/species-bank/sbank-treatment2.pl?id=5720.Consulta noviembre 2018

Bahamóndez, C.; Ferrando, M.; Martin, M. y Pinilla, J. C., 1995. Determinación de Funciones de Volumen para Eucalipto. Antecedentes Biométricos y Modelos de Apoyo a la Gestión y Manejo Racional del Eucalipto. FONDEFINFOR, Chile.

Martin, M. y Bahamóndez, C., 2000. Determinación de Funciones de Volumen para Eucalipto. Documento de Trabajo PE - 01. Proyecto Escalamiento de las Técnicas de Producción y Manejo de las Principales Especies de Eucalipto Plantadas en Chile. Valdivia, Chile. 10p.

Friedl, R. A; Correa, M. A.; Toloza, R.; Hennig, H.; Termachuka, M.; Martínez, C.; Osorio, M. y Parodi, G., 2010. Ajuste de un modelo para obtención de volumen para árboles individuales de Acacia mearnsii De Wild (Primera Aproximación). 14 ${ }^{\text {as }}$ Jornadas Técnicas Forestales y Ambientales. Facultad de Ciencias Forestales, UNaM - EEA Montecarlo, INTA. 10, 11 y 12 de junio de 2010 -Eldorado, Misiones, Argentina

INFOR, 1999. Incorporación de especies del género Acacia a la producción forestal chilena. Informe de avance. INFOR - CORFO. Concepción. 126 p.

INFOR, 2018. Anuario Forestal 2018. Instituto Forestal, Chile. Boletín Estadístico N 163. P. 177.

Kannegiesser, U., 1990. Apuntes sobre algunas acacias australianas: 1. Acacia mearnsii de Willd. Ciencia e Investigación Forestal, Volumen 4 (2) diciembre 1990. Instituto Forestal - Chile. pp.198-212.

NAS, 1980. Firewood crops. Shrubs and tree species for energy production. Vol. 1. Washington D.C. National Academy of Sciences, National Academy Press. 237 p.

Pinilla, Juan Carlos, 2000. Descripción y antecedentes básicos sobre Acacia dealbata, Acacia melanoxylon y Acacia mearnsii. Revisión bibliográfica; Instituto Forestal, (Santiago, Chile). Informe Técnico N¹47. Santiago. INFOR, 2000. 49 p.

Pinilla, J. C.; Molina, M. P.; Luengo, K. y Navarrete, M., 2015. Evaluación a los tres años de edad de un 
ensayo de progenies de Acacia mearnsii de Wild establecido en Los Sauces, Región de la Araucanía. En: Ciencia e Investigación Forestal, CIFOR, V.21(1), Pág.: 37-68.

Pinilla, J. C.; Luengo, K. y Navarrete, M., 2018. Crecimiento de Acacia mearnsii De Wild en Chile. Primeros Antecedentes. En Actas VII Congreso Forestal Latinoamericano. Vitoria, Brasil, 12 a 15 de junio 2018.

Prodan, M.; Peters, R.; Cox, F. y Real, P., 1997. Mensura Forestal. Proyecto IICA/GTZ sobre Agricultura, Recursos Naturales y Desarrollo. San José CR.

Schneider, P.; Dimas, F.; Guimarães, C. y Mayer, J. ,2000. Crescimento da acácia-negra, Acacia mearnsii De Wild em diferentes espaçamentos. Revista Ciência Florestal, Santa Maria, v. 10, n. 2, p. 101-112 
